\title{
Tratamientos prostodónticos en adultos mayores realizados en un centro dental especializado entre los años 2016 y 2018
}

\author{
Prosthodontic treatments in older adults performed in a specialized dental center between 2016 and 2018
}

\author{
Sandro Alexander Lévano Loayza ${ }^{1, a, b}$, Gabriella Jackeline Rodriguez Ramirez ${ }^{1, \mathrm{c}}$, Lillie Elizabeth Abanto Silva ${ }^{2, d, \mathrm{e}}$
}

\begin{abstract}
RESUMEN
Los adultos mayores son más propensos a sufrir enfermedades y comorbilidades que afectan su estado bucal, por lo que necesitan de tratamientos prostodónticos acondicionados a su estado sistémico para devolverles la función masticatoria, estética y armonía oral. No obstante, la pobre cultura de prevención y la falta de profesionales especializados en su atención dificulta en gran medida esta labor. Objetivo: Determinar los tratamientos prostodónticos en pacientes adultos mayores realizados en el Servicio de Estomatología en Pacientes Especiales del Centro Dental Docente Cayetano Heredia desde abril de 2016 hasta diciembre de 2018. Material y métodos: Se realizó un estudio observacional, descriptivo, transversal y retrospectivo en una muestra de 144 adultos mayores con 200 tratamientos realizados. Se consideraron variables demográficas como sexo, grupo etario y procedencia. Se elaboró una base de datos, y posteriormente tablas de contenido para el análisis de sus resultados. Resultados: La prótesis parcial removible fue el tratamiento más realizado para ambos sexos, 39,8\% ( $\mathrm{n}=57$ ) para el sexo femenino y $38,6 \%(\mathrm{n}=22)$ para el sexo masculino. De acuerdo al grupo etario, los viejos-viejos fueron los pacientes que más prótesis parcial removible se realizaron con 40,19\% $(\mathrm{n}=43)$. La mayoría de los pacientes provenientes de Lima Norte se realizaron el tratamiento de prótesis parcial removible con $41.96 \%(\mathrm{n}=47)$. Conclusiones: Las prótesis removibles predominaron más que las fijas, siendo la prótesis parcial removible la que más se realizó. También se evidencio que el sexo femenino recibió más tratamientos prostodónticos y que el lugar de procedencia que más se registro fue el de Lima Norte, posiblemente por la cercanía al centro dental.
\end{abstract}

PALABRAS CLAVE: Adulto mayor, prótesis dental, odontología geriátrica.

\section{SUMMARY}

Older adults are more prone to suffer diseases and comorbidities that affect their oral condition, so they need prosthodontic treatments conditioned to their systemic state to restore their masticatory, aesthetic and oral harmony function. However, the poor culture of prevention and the lack of specialized professionals in their care greatly hinders this work. Objective: To determine the prosthodontic treatments in older adults performed in the Service of Estomatología in Pacientes Especiales of the Centro Dental Docente Cayetano Heredia from april 2016 to december 2018. Material and methods: An observational, descriptive, cross-sectional, and retrospective study was carried out in a sample of 144 older adults with 200 treatments performed. Demographic variables such as sex, age group and origin wereconsidered. A database was developed, and later tables of contents for the analysis of its results. Results: removable partial denture was the most performed treatment for both sexes, $39.8 \%(\mathrm{n}=57)$ for the female sex and $38.6 \%(\mathrm{n}=22)$ for the male sex. According to the age group, the oldold were the patients who performed the most removable partial denture with $40.19 \%(n=43)$. Most of the patients from Lima Norte underwent removable partial denturetreatment with $41.96 \%(n=47)$. Conclusions: The removable prostheses predominated more than the fixed ones, being the removable partial denture the one that was performed the most. It was also evidenced that the female sex received more prosthodontic treatments and that the place of origin that was most recorded was Lima Norte, possibly due to its proximity to the dental center.

KEYWORDS: Senior adult, dental prosthesis, geriatric dentistry.

Facultad de Estomatología, Universidad Peruana Cayetano Heredia. Lima, Perú.

Departamento Académico de Medicina y Cirugía Bucomaxilofacial, Facultad de Estomatología, Universidad Peruana Cayetano Heredia. Lima, Perú.

Cirujano Dentista

Diplomado en Cirugía Oral

Bachiller en Estomatología

Especialista en Estomatología de Pacientes Especiales

Docente 


\section{INTRODUCCIÓN}

Se considera Adulto Mayor (AM) a la persona mayor a 60 años de edad (1), que experimenta cambios a nivel fisiológico, psicológico y social, y que usualmente tiene mayor facilidad de enfermarse y presentar trastornos de comportamiento (2). Los AM se pueden clasificar por grupos de edad, siendo estos los viejosjóvenes (60 a 74 años), los viejos-viejos (75 a 89 años) y los viejos-longevos (90 a más años) (3).

A nivel general, los AM sufren múltiples cambios como las alteraciones en todos los aparatos y sistemas del cuerpo (4-6). También consumen múltiples fármacos (polifarmacia), por tal motivo es necesario evaluar factores como toxicidad y sensibilidad a ciertos medicamentos para evitar efectos adversos (7).

En la cavidad oral también se presentan diversas enfermedades como la caries dental, enfermedad periodontal y lesiones en la mucosa oral (benignas, malignas, y de origen fúngico). Asimismo, se presentan cambios sensoriales en las zonas gustativas de la lengua y una disminución del flujo salival conocido como xerostomía. También se pueden presentar lesiones como el épulis fisurado y la úlcera traumática producto del uso de prótesis mal adaptadas, o queilitis angular y candidiasis oral, generalmente asociado a infecciones fúngicas o deficiencias vitamínicas por una patología pre existente (8). Por ello es importante conocer si el AM presenta una comorbilidad que afecte su estado bucal, como es el caso de pacientes con alteraciones en la presión arterial, diabetes mellitus, pacientes con antecedentes de accidente cerebrovascular, con terapia anticoagulante, con enfermedad pulmonar obstructiva crónica, entre otras enfermedades $(9,10)$.

A fin de darles una mejor calidad de vida, se pueden realizar diferentes tipos de tratamientos odontológicos como los prostodónticos, los cuales deben estar sujetos a las condiciones sistémicas del paciente para devolverle la función masticatoria, estética y armonía oral mediante la utilización de prótesis dentales fijas o removibles (11).

En el Perú la atención odontológica adecuada a este tipo de pacientes sigue siendo una gran problemática por la pobre cultura de prevención y la inexistencia de la especialidad de Odontogeriatría que forme profesionales especializados en su atención, así como también la realización de tratamientos odontológicos de acuerdo a su condición sistémica. Por lo cual esta investigación brinda un panorama general y datos concisos de los tratamientos prostodónticos en AM permitiéndonos estar más preparados para las futuras rehabilitaciones. Además, proporciona un diagnostico situacional de la realidad que los AM afrontan por la pérdida de piezas dentarias, así como también permite evaluar la condición de indiferencia del AM en el Perú, el cual muchas veces es dejado de lado y no se le da el tratamiento idóneo por su longevidad. Por ello este estudio ocasiona un gran impacto en la sociedad ya que puede ser el inicio de un cambio mediante la creación de políticas públicas que mejoren la atención de estos pacientes, en el ámbito preventivo, correctivo o de mantenimiento donde se busque su independencia funcional y consecuentemente una mejor la calidad de vida.

El objetivo de este estudio fue determinar los tratamientos prostodónticos en pacientes adultos mayores, realizados en el Servicio de Estomatología en Pacientes Especiales del Centro Dental Docente Cayetano Heredia desde abril de 2016 hasta diciembre de 2018.

\section{MATERIAL Y MÉTODOS}

El estudio fue observacional, descriptivo, transversal y retrospectivo. Se obtuvo la aprobación del Comité Institucional de Ética en Investigación de la Universidad Peruana Cayetano Heredia y la autorización del Centro Dental Docente de la Universidad Peruana Cayetano Heredia (CDCUPCH) para acceder a la base de datos de los pacientes del Servicio de Estomatología en Pacientes Especiales (EPE).

La variable principal del estudio fue determinar los tratamientos prostodónticos en pacientes AM, para lo cual se procedió a la búsqueda de la atención en pacientes AM que cumplieran los criterios de inclusión (hayan recibido y culminado algún tipo de tratamiento prostodóntico) y exclusión (no culminaron su tratamiento protésico, pacientes adultos mayores con alguna enfermedad sistémica no controlada o con incapacidad física o mental para poder realizarle algún tratamiento odontológico y pacientes adultos mayores que necesiten sedación), en el periodo de abril 2016 a diciembre 2018. También se consideraron variables 
demográficas como sexo, grupo etario y procedencia (Distribución de distritos de Lima Metropolitana según el Instituto Nacional de Estadística e Informática) (tabla 1). Se elaboró una base de datos, y posteriormente tablas de contenido para el análisis de sus resultados. Finalmente se procedió al análisis de los resultados de la base de datos para obtener los registros finales de las respuestas halladas. Luego se evaluó de acuerdo a las variables del estudio. Para la obtención de los resultados se utilizó chi cuadrado, y el nivel de significancia se fijó en un 95\%. El programa estadístico usado fue el SPSS versión 24.0.

Tabla 1. Distribución de distritos de Lima Metropolitana según INEI

\begin{tabular}{|c|c|}
\hline ÁREAS DE LIMA & DISTRITOS \\
\hline Lima Norte & $\begin{array}{c}\text { San Juan de Lurigancho } \\
\text { San Martín de Porres } \\
\text { Comas } \\
\text { Los Olivos } \\
\text { Puente Piedra } \\
\text { Carabayllo } \\
\text { Independencia } \\
\text { Rimac } \\
\text { Ancón } \\
\text { Santa Rosa }\end{array}$ \\
\hline Lima Centro & $\begin{array}{c}\text { Cercado de Lima } \\
\text { La Victoria } \\
\text { San Miguel } \\
\text { San Borja } \\
\text { Surquillo } \\
\text { Miraflores } \\
\text { Breña } \\
\text { Pueblo Libre } \\
\text { Jesús María } \\
\text { San Isidro } \\
\text { Lince } \\
\text { San Luis } \\
\text { Magdalena }\end{array}$ \\
\hline Lima Este & $\begin{array}{c}\text { Ate } \\
\text { Santa Anita } \\
\text { El Agustino } \\
\text { San Juan de Lurigancho } \\
\text { La Molina } \\
\text { Chaclacayo } \\
\text { Cineguilla }\end{array}$ \\
\hline Lima Sur & $\begin{array}{c}\text { Villa El Salvador } \\
\text { Villa María Del Triunfo } \\
\text { San Juan de Miraflores } \\
\text { Surco } \\
\text { Chorrillos } \\
\text { Pachacamac } \\
\text { Lurín } \\
\text { Barranco } \\
\text { Pucusana } \\
\text { San Bartolo } \\
\text { Punta Hermosa } \\
\text { Punta Negra } \\
\text { Santa María del Mar }\end{array}$ \\
\hline P.C. Callao & $\begin{array}{c}\text { Callao } \\
\text { Ventanilla } \\
\text { Bellavista } \\
\text { La Perla } \\
\text { Carmen de La Legua } \\
\text { La Punta }\end{array}$ \\
\hline Provincia & - \\
\hline
\end{tabular}




\section{RESULTADOS}

De una población de 1271 pacientes se obtuvo una muestra de 144 pacientes AM, de los cuales $69,4 \%$ $(\mathrm{n}=100)$ fueron del sexo femenino y $30,5 \%(\mathrm{n}=44)$ del sexo masculino. Según edad el promedio de edad de los participantes fue de 75,53 $( \pm 7,50)$, siendo los de mayor número los viejos-viejos con $52,08 \%$ $(\mathrm{n}=74)$ y en menor número los viejos-longevos con $3.47 \%(\mathrm{n}=5)$. Según procedencia, se evidenció que la mayoría proviene de Lima Norte $53,47 \%(\mathrm{n}=77)$ y la menor cantidad de pacientes provinieron de Lima Sur $1,39 \%(\mathrm{n}=2)($ tabla 2$)$

Se realizaron 200 tratamientos protésicos entre incrustaciones, coronas, puentes, prótesis parcial removible (PPR) y prótesis totales (PT), de los cuales la mayor cantidad fueron las PPR con 39,5\% $(n=79)$, las PT con $35,5 \%(\mathrm{n}=71)$ y en menor cantidad las prótesis fijas, incrustaciones $6 \%(\mathrm{n}=12)$ y puentes $4 \%$ $(\mathrm{n}=8)$. Además, se observó el número de tratamiento según su tipo, en donde se evidenció que la mayoría de pacientes solo se realizó una PPR 68,35\% $(\mathrm{n}=54)$ (tabla 3).

De acuerdo a tratamientos según sexo, se evidenció que el sexo femenino fue el que más tratamientos protésicos se realizó con 71,5\% ( $\mathrm{n}=143)$. La PPR predomino para ambos sexos, $39,8 \%(\mathrm{n}=57)$ para el sexo femenino y $38,6 \%(\mathrm{n}=22)$ para el sexo masculino. Los menos realizados fueron los puentes con $3,51 \%$ $(\mathrm{n}=2)$ y $4,2 \%(\mathrm{n}=6)$ para el sexo masculino y femenino respectivamente (tabla 4 ).

Con respecto a tratamientos según edad, se observó que el grupo de viejos-viejos fue el que más tratamientos prostodónticos se realizó con 53,5\% $(\mathrm{n}=107)$, destacando la PPR con 40,19\% ( $\mathrm{n}=43)$. El puente junto a las incrustaciones fueron los menos realizados con $3,74 \%(n=4)$. Por otro lado, los viejos-jóvenes, se realizaron el $42,5 \%(n=85)$ de los tratamientos, dentro de los cuales la PPR nuevamente predomino con $40 \%(n=34)$ y el puente fue el menos confeccionado con 4,71\% (n=4). Por último, los viejos-longevos se realizaron el $4 \%(n=8)$ de los tratamientos, siendo la PT la más elaborada con $62,5 \%(n=5)$ y no se registraron tratamientos de incrustaciones y puentes (tabla 5)

De acuerdo a procedencia se evidenció que la mayoría de pacientes provenientes de Lima Norte se realizaron PPR con 41,96\% $(n=47)$, los tratamientos menos realizados en la misma área fueron los puentes e incrustaciones con 4,46\% $(n=5)$ cada uno. En Lima Centro los tratamientos más realizados fueron las PPR y los menos realizados fueron los puentes. En Lima Este la mayor cantidad de tratamientos realizados fueron las coronas y las prótesis totales, no se realizaron tratamientos de incrustaciones ni puentes. En Lima Sur, se registraron la misma cantidad de tratamientos realizados para coronas, puentes y prótesis totales y no se realizaron PPR ni incrustaciones. En la P.C. del Callao, en mayor cantidad se realizó PT y en menor cantidad coronas. Y por último la mayoría de pacientes provenientes de provincia se realizaron prótesis removibles con $39,47 \%(n=15)$ para PPR y PT cada uno, y en menor cantidad se realizaron puentes con $2,63 \%(n=1)$ (tabla 6$)$.

La última tabla muestra la relación entre los tipos de tratamientos según sexo y lugar de procedencia, en donde se observó que para el sexo masculino la PPR fue el tratamiento más realizado con $38,60 \%$ $(n=22)$, como se mencionó anteriormente en la tabla 4 , teniendo como principal lugar de procedencia Lima Norte para este tipo de tratamiento con $45,45 \%$ $(\mathrm{n}=10)$, luego le sigue Lima Centro y provincia con $22,73 \%(n=5)$. Por otro lado, el tratamiento menos realizado en el sexo masculino fue la prótesis fija, específicamente los puentes y según su procedencia fue en Lima Norte donde se realizó en mayor cantidad este tipo de procedimiento. Para el sexo femenino las PPR también fueron los tratamientos más realizados, dentro del mismo, se observó que en su mayoría los pacientes que se realizaron dicho tratamiento provienen de Lima Norte con un 64,91\% $(n=37)$, seguidos de los provenientes de provincia. La PT fue el segundo tratamiento más realizado con $35,66 \%$ $(\mathrm{n}=51)$, dentro del cual en su mayoría la procedencia era de Lima Norte con 54,90\% $(n=28)$ seguido de provincia con $21,57 \%(\mathrm{n}=11)$, y por último, los tratamientos menos comunes fueron los puentes con 4,20\% $(\mathrm{n}=6)$ provenientes de Lima Norte (tabla 7). 
Tabla 2. Características de los pacientes AM atendidos en el Servicio de EPE del CDC-UPCH

\begin{tabular}{llcc}
\hline & Variables & n & \% \\
\hline Sexo & & & \\
& Masculino & 44 & 30.56 \\
& Femenino & 100 & 69.44
\end{tabular}

Edad (Promedio=75.53, Desviación estándar=7.50)

$\begin{array}{lcc}\text { Viejos-jóvenes } & 64 & 44.44 \\ \text { Viejos-viejos } & 75 & 52.08 \\ \text { Viejos-longevos } & 5 & 3.47\end{array}$

Procedencia

$\begin{array}{lcc}\text { Lima Norte } & 77 & 53.47 \\ \text { Lima Centro } & 20 & 13.89 \\ \text { Lima Este } & 8 & 5.56 \\ \text { Lima Sur } & 2 & 1.39 \\ \text { P. C. del Callao } & 9 & 6.25 \\ \text { Provincias } & 28 & 19.44\end{array}$

$\mathrm{n}$ : Frecuencia absoluta.

$\%$ : Frecuencia relativa.

Tabla 3. Tipo y número de tratamientos prostodóntico en los pacientes AM realizados en el Servicio de EPE del CDC-UPCH

\begin{tabular}{|c|c|c|c|c|c|c|c|c|c|c|c|c|c|c|c|c|c|c|}
\hline $\begin{array}{c}\text { Tipo de } \\
\text { tratamiento }\end{array}$ & $\mathrm{n}$ & $\%$ & $\begin{array}{c}\text { Número } \\
\text { de } \\
\text { tratamientos }\end{array}$ & & & & & & & & & & & & & & & \\
\hline & & & 1 & & 2 & & 3 & & 4 & & 5 & & 6 & & 7 & & 9 & \\
\hline & & & $\mathbf{n}$ & $\%$ & $\mathrm{n}$ & $\%$ & $\mathrm{n}$ & $\%$ & $\mathrm{n}$ & $\%$ & $\mathrm{n}$ & $\%$ & $\mathrm{n}$ & $\%$ & $\mathrm{n}$ & $\%$ & n & $\%$ \\
\hline Incrustaciones & 12 & 6.00 & 8 & 66.67 & 2 & 16.67 & 1 & 8.33 & 0 & 0.00 & 1 & 8.33 & 0 & 0.00 & 0 & 0.00 & 0 & 0.00 \\
\hline Coronas & 30 & 15.00 & 11 & 36.67 & 8 & 26.67 & 4 & 13.33 & 2 & 6.67 & 1 & 3.33 & 2 & 6.67 & 1 & 3.33 & 1 & 3.33 \\
\hline Puentes & 8 & 4.00 & 8 & 100.00 & 0 & 0.00 & 0 & 0.00 & 0 & 0.00 & 0 & 0.00 & 0 & 0.00 & 0 & 0.00 & 0 & 0.00 \\
\hline PPR & 79 & 39.50 & 54 & 68.35 & 25 & 31.65 & - & - & - & - & - & - & - & - & - & - & - & - \\
\hline P. Total & 71 & 35.50 & 40 & 56.34 & 31 & 43.66 & - & - & - & - & - & - & - & - & - & - & - & - \\
\hline Total & 200 & 100.00 & 121 & 60.50 & 66 & 33.00 & 5 & 2.50 & 2 & 1.00 & 2 & 1.00 & 2 & 1.00 & 1 & 0.50 & 1 & 0.50 \\
\hline
\end{tabular}

n: Frecuencia absoluta.

$\%$ : Frecuencia relativa.

Prueba de Chi-cuadrado corregido por Yates: Tipo de tratamiento vs. $\mathrm{N}^{\circ}$ de tratamientos $(\mathrm{p}=<0.01)$. 
Tabla 4. Tipo y número de tratamientos prostodónticos según género en pacientes AM realizados en el Servicio de EPE del CDC-UPCH

\begin{tabular}{|c|c|c|c|c|c|c|c|c|c|c|c|c|c|c|c|c|c|c|c|}
\hline \multirow{3}{*}{ Sexo } & \multirow{3}{*}{$\begin{array}{c}\text { Tipo de } \\
\text { tratamiento }\end{array}$} & \multirow{3}{*}{ n } & \multirow{3}{*}{$\%$} & \multicolumn{16}{|c|}{ Número de tratamientos } \\
\hline & & & & \multicolumn{2}{|c|}{1} & \multicolumn{2}{|l|}{2} & \multicolumn{2}{|l|}{3} & \multicolumn{2}{|c|}{4} & \multicolumn{2}{|c|}{5} & \multicolumn{2}{|l|}{6} & \multicolumn{2}{|l|}{7} & \multicolumn{2}{|r|}{9} \\
\hline & & & & $\mathbf{n}$ & $\%$ & $\mathbf{n}$ & $\%$ & $\mathbf{n}$ & $\%$ & $\mathbf{n}$ & $\%$ & $\mathbf{n}$ & $\%$ & $\mathbf{n}$ & $\%$ & $\mathrm{n}$ & $\%$ & $\mathbf{n}$ & $\%$ \\
\hline \multicolumn{20}{|c|}{ Masculino } \\
\hline & $\begin{array}{l}\text { Incrusta- } \\
\text { ciones }\end{array}$ & 3 & 5.26 & 2 & 66.67 & 1 & 33.33 & 0 & 0.00 & 0 & 0.00 & 0 & 0.00 & 0 & 0.00 & 0 & 0.00 & 0 & 0.00 \\
\hline & Coronas & 10 & 17.54 & 3 & 30.00 & 5 & 50.00 & 1 & 10.00 & 1 & 10.00 & 0 & 0.00 & 0 & 0.00 & 0 & 0.00 & 0 & 0.00 \\
\hline & Puentes & 2 & 3.51 & 2 & 100.00 & 0 & 0.00 & 0 & 0.00 & 0 & 0.00 & 0 & 0.00 & 0 & 0.00 & 0 & 0.00 & 0 & 0.00 \\
\hline & PPR & 22 & 38.60 & 15 & 68.18 & 7 & 31.82 & - & - & - & - & - & - & - & - & - & - & - & - \\
\hline & P. Total & 20 & 35.09 & 10 & 50.00 & 10 & 50.00 & - & - & - & - & - & - & - & - & - & - & - & - \\
\hline & Total & 57 & 100.00 & 32 & 56.14 & 23 & 40.35 & 1 & 1.75 & 1 & 1.75 & 0 & 0.00 & 0 & 0.00 & 0 & 0.00 & 0 & 0.00 \\
\hline \multicolumn{20}{|c|}{ Femenino } \\
\hline & $\begin{array}{l}\text { Incrusta- } \\
\text { ciones }\end{array}$ & 9 & 6.29 & 6 & 66.67 & 1 & 11.11 & 1 & 11.11 & 0 & 0.00 & 1 & 11.11 & 0 & 0.00 & 0 & 0.00 & 0 & 0.00 \\
\hline & Coronas & 20 & 13.99 & 8 & 40.00 & 3 & 15.00 & 3 & 15.00 & 1 & 5.00 & 1 & 5.00 & 2 & 10.00 & 1 & 5.00 & 1 & 5.00 \\
\hline & Puentes & 6 & 4.20 & 6 & 100.00 & 0 & 0.00 & 0 & 0.00 & 0 & 0.00 & 0 & 0.00 & 0 & 0.00 & 0 & 0.00 & 0 & 0.00 \\
\hline & PPR & 57 & 39.86 & 39 & 68.42 & 18 & 31.58 & - & - & - & - & - & - & - & - & - & - & - & - \\
\hline & P. Total & 51 & 35.66 & 30 & 58.82 & 21 & 41.18 & - & - & - & - & - & - & - & - & - & - & - & - \\
\hline & Total & 143 & 100.00 & 89 & 62.24 & 43 & 30.07 & 4 & 2.80 & 1 & 0.70 & 2 & 1.40 & 2 & 1.40 & 1 & 0.70 & 1 & 0.70 \\
\hline
\end{tabular}

n: Frecuencia absoluta.

$\%$ : Frecuencia relativa.

Prueba de Chi-cuadrado: Sexo vs. Tipo de tratamiento $(\mathrm{p}=0.98)$ y Sexo

vs. $\mathrm{N}^{\circ}$ de tratamientos $(\mathrm{p}=0.71)$. 
Tabla 5. Tipo y número de tratamientos prostodónticos según edad en pacientes AM realizados en el Servicio de EPE del CDC-UPCH

\begin{tabular}{|c|c|c|c|c|c|c|c|c|c|c|c|c|c|c|c|c|c|c|c|}
\hline \multirow{3}{*}{ Edad } & \multirow{3}{*}{$\begin{array}{c}\text { Tipo de } \\
\text { tratamiento }\end{array}$} & \multirow{3}{*}{ n } & \multirow{3}{*}{$\%$} & \multicolumn{16}{|c|}{ Número de tratamientos } \\
\hline & & & & \multicolumn{2}{|c|}{1} & \multicolumn{2}{|c|}{2} & \multicolumn{2}{|c|}{3} & \multicolumn{2}{|c|}{4} & \multicolumn{2}{|c|}{5} & \multicolumn{2}{|c|}{6} & \multicolumn{2}{|c|}{7} & \multicolumn{2}{|c|}{9} \\
\hline & & & & $\mathbf{n}$ & $\%$ & $\mathbf{n}$ & $\%$ & $\mathbf{n}$ & $\%$ & $\mathbf{n}$ & $\%$ & $\mathbf{n}$ & $\%$ & $\mathbf{n}$ & $\%$ & $\mathbf{n}$ & $\%$ & $\mathbf{n}$ & $\%$ \\
\hline
\end{tabular}

Viejos-jóvenes

$\begin{array}{lcccccccccccccccccc}\text { Incrustaciones } & 8 & 9.41 & 5 & 62.50 & 2 & 25.00 & 0 & 0.00 & 0 & 0.00 & 1 & 12.50 & 0 & 0.00 & 0 & 0.00 & 0 & 0.00 \\ \text { Coronas } & 13 & 15.29 & 7 & 53.85 & 2 & 15.38 & 1 & 7.69 & 1 & 7.69 & 0 & 0.00 & 1 & 7.69 & 1 & 7.69 & 0 & 0.00 \\ \text { Puentes } & 4 & 4.71 & 4 & 100.00 & 0 & 0.00 & 0 & 0.00 & 0 & 0.00 & 0 & 0.00 & 0 & 0.00 & 0 & 0.00 & 0 & 0.00 \\ \text { PPR } & 34 & 40.00 & 24 & 70.59 & 10 & 29.41 & - & - & - & - & - & - & - & - & - & - & - \\ \text { P. Total } & 26 & 30.59 & 13 & 50.00 & 13 & 50.00 & - & - & - & - & - & - & - & - & - & - & - & - \\ & & & & & & & & & & & & & & & & \\ \text { Total } & 85 & 100.00 & 53 & 62.35 & 27 & 31.76 & 1 & 1.18 & 1 & 1.18 & 1 & 1.18 & 1 & 1.18 & 1 & 1.18 & 0 & 0.00\end{array}$

Viejos-viejos

$\begin{array}{lcccccccccccccccccc}\text { Incrustaciones } & 4 & 3.74 & 3 & 75.00 & 0 & 0.00 & 1 & 25.00 & 0 & 0.00 & 0 & 0.00 & 0 & 0.00 & 0 & 0.00 & 0 & 0.00 \\ \text { Coronas } & 16 & 14.95 & 4 & 25.00 & 5 & 31.25 & 3 & 18.75 & 1 & 6.25 & 1 & 6.25 & 1 & 6.25 & 0 & 0.00 & 1 & 6.25 \\ \text { Puentes } & 4 & 3.74 & 4 & 100.00 & 0 & 0.00 & 0 & 0.00 & 0 & 0.00 & 0 & 0.00 & 0 & 0.00 & 0 & 0.00 & 0 & 0.00 \\ \text { PPR } & 43 & 40.19 & 28 & 65.12 & 15 & 34.88 & - & - & - & - & - & - & - & - & - & - & - & - \\ \text { P. Total } & 40 & 37.38 & 23 & 57.50 & 17 & 42.50 & - & - & - & - & - & - & - & - & - & - & - & - \\ & & & & & & & & & & & & & & & & \\ \text { Total } & 107 & 100.00 & 62 & 57.94 & 37 & 34.58 & 4 & 3.74 & 1 & 0.93 & 1 & 0.93 & 1 & 0.93 & 0 & 0.00 & 1 & 0.93\end{array}$

Viejos-longevos

\begin{tabular}{|c|c|c|c|c|c|c|c|c|c|c|c|c|c|c|c|c|c|c|}
\hline Coronas & 1 & 12.50 & 0 & 0.00 & 1 & 100.00 & 0 & 0.00 & 0 & 0.00 & 0 & 0.00 & 0 & 0.00 & 0 & 0.00 & 0 & 0.00 \\
\hline PPR & 2 & 25.00 & 2 & 100.00 & 0 & 0.00 & - & - & - & - & - & - & - & - & - & - & - & - \\
\hline P. Total & 5 & 62.50 & 4 & 80.00 & 1 & 20.00 & - & - & - & - & - & - & - & - & - & - & - & - \\
\hline Total & 8 & 100.00 & 6 & 75.00 & 2 & 25.00 & 0 & 0.00 & 0 & 0.00 & 0 & 0.00 & 0 & 0.00 & 0 & 0.00 & 0 & 0.00 \\
\hline
\end{tabular}

n: Frecuencia absoluta.

$\%$ : Frecuencia relativa.

Prueba de Chi-cuadrado: Edad vs. Tipo de tratamiento $(\mathrm{p}=0.61)$ y Edad vs. $\mathrm{N}^{\circ}$ de tratamientos $(\mathrm{p}=0.99)$. 
Tabla 6. Tipo y número de tratamientos prostodónticos según procedencia en pacientes AM realizados en el Servicio de EPE del CDC-UPCH

\begin{tabular}{|c|c|c|c|c|c|c|c|c|c|c|c|c|c|c|c|c|c|c|c|}
\hline \multirow{3}{*}{ Procedencia } & \multirow{3}{*}{$\begin{array}{c}\text { Tipo de } \\
\text { tratamiento }\end{array}$} & \multirow{3}{*}{$\mathbf{n}$} & \multirow{3}{*}{$\%$} & \multicolumn{16}{|c|}{ Número de tratamientos } \\
\hline & & & & \multicolumn{2}{|r|}{1} & \multicolumn{2}{|r|}{2} & \multicolumn{2}{|c|}{3} & \multicolumn{2}{|c|}{4} & \multicolumn{2}{|c|}{5} & \multicolumn{2}{|c|}{6} & \multicolumn{2}{|c|}{7} & \multicolumn{2}{|c|}{9} \\
\hline & & & & $\mathbf{n}$ & $\%$ & $\mathbf{n}$ & $\%$ & $\mathbf{n}$ & $\%$ & $\mathbf{n}$ & $\%$ & $\mathbf{n}$ & $\%$ & $\mathbf{n}$ & $\%$ & $\mathbf{n}$ & $\%$ & $\mathbf{n}$ & $\%$ \\
\hline \multicolumn{20}{|l|}{ Lima Norte } \\
\hline & Incrustaciones & 5 & 4.46 & 3 & 60.00 & 1 & 20.00 & 1 & 20.00 & 0 & 0.00 & 0 & 0.00 & 0 & 0.00 & 0 & 0.00 & 0 & 0.00 \\
\hline & Coronas & 17 & 15.18 & 6 & 35.29 & 2 & 11.76 & 3 & 17.65 & 2 & 11.76 & 1 & 5.88 & 2 & 11.76 & 1 & 5.88 & 0 & 0.00 \\
\hline & Puentes & 5 & 4.46 & 5 & 100.00 & 0 & 0.00 & 0 & 0.00 & 0 & 0.00 & 0 & 0.00 & 0 & 0.00 & 0 & 0.00 & 0 & 0.00 \\
\hline & PPR & 47 & 41.96 & 32 & 68.09 & 15 & 31.91 & - & - & - & - & - & - & - & - & - & - & - & - \\
\hline & P. Total & 38 & 33.93 & 21 & 55.26 & 17 & 44.74 & - & - & - & - & - & - & - & - & - & - & - & - \\
\hline & Total & 112 & 100.00 & 67 & 59.82 & 35 & 31.25 & 4 & 3.57 & 2 & 1.79 & 1 & 0.89 & 2 & 1.79 & 1 & 0.89 & 0 & 0.00 \\
\hline \multicolumn{20}{|l|}{ Lima Centro } \\
\hline & Incrustaciones & 2 & 7.41 & 2 & 100.00 & 0 & 0.00 & 0 & 0.00 & 0 & 0.00 & 0 & 0.00 & 0 & 0.00 & 0 & 0.00 & 0 & 0.00 \\
\hline & Coronas & 2 & 7.41 & 1 & 50.00 & 0 & 0.00 & 0 & 0.00 & 0 & 0.00 & 0 & 0.00 & 0 & 0.00 & 0 & 0.00 & 1 & 50.00 \\
\hline & Puentes & 1 & 3.70 & 1 & 100.00 & 0 & 0.00 & 0 & 0.00 & 0 & 0.00 & 0 & 0.00 & 0 & 0.00 & 0 & 0.00 & 0 & 0.00 \\
\hline & PPR & 13 & 48.15 & 8 & 61.54 & 5 & 38.46 & - & - & - & - & - & - & - & - & - & - & - & - \\
\hline & P. Total & 9 & 33.33 & 7 & 77.78 & 2 & 22.22 & - & - & - & - & - & - & - & - & - & - & - & - \\
\hline & Total & 27 & 100.00 & 19 & 70.37 & 7 & 25.93 & 0 & 0.00 & 0 & 0.00 & 0 & 0.00 & 0 & 0.00 & 0 & 0.00 & 1 & 3.70 \\
\hline \multicolumn{20}{|l|}{ Lima Este } \\
\hline & Coronas & 4 & 40.00 & 0 & 0.00 & 3 & 75.00 & 1 & 25.00 & 0 & 0.00 & 0 & 0.00 & 0 & 0.00 & 0 & 0.00 & 0 & 0.00 \\
\hline & PPR & 2 & 20.00 & 2 & 100.00 & 0 & 0.00 & - & - & - & - & - & - & - & - & - & - & - & - \\
\hline & P. Total & 4 & 40.00 & 0 & 0.00 & 4 & 100.00 & - & - & - & - & - & - & - & - & - & - & - & - \\
\hline & Total & 10 & 100.00 & 2 & 20.00 & 7 & 70.00 & 1 & 10.00 & 0 & 0.00 & 0 & 0.00 & 0 & 0.00 & 0 & 0.00 & 0 & 0.00 \\
\hline \multicolumn{20}{|l|}{ Lima Sur } \\
\hline & Coronas & 1 & 33.33 & 0 & 0.00 & 1 & 100.00 & 0 & 0.00 & 0 & 0.00 & 0 & 0.00 & 0 & 0.00 & 0 & 0.00 & 0 & 0.00 \\
\hline & Puentes & 1 & 33.33 & 1 & 100.00 & 0 & 0.00 & 0 & 0.00 & 0 & 0.00 & 0 & 0.00 & 0 & 0.00 & 0 & 0.00 & 0 & 0.00 \\
\hline & P. Total & 1 & 33.33 & 1 & 100.00 & 0 & 0.00 & - & - & - & - & - & - & - & - & - & - & - & - \\
\hline & Total & 3 & 100.00 & 2 & 66.67 & 1 & 33.33 & 0 & 0.00 & 0 & 0.00 & 0 & 0.00 & 0 & 0.00 & 0 & 0.00 & 0 & 0.00 \\
\hline \multicolumn{20}{|l|}{ P. C. del Callao } \\
\hline & Incrustaciones & 3 & 30.00 & 1 & 33.33 & 1 & 33.33 & 0 & 0.00 & 0 & 0.00 & 1 & 33.33 & 0 & 0.00 & 0 & 0.00 & 0 & 0.00 \\
\hline & Coronas & 1 & 10.00 & 1 & 100.00 & 0 & 0.00 & 0 & 0.00 & 0 & 0.00 & 0 & 0.00 & 0 & 0.00 & 0 & 0.00 & 0 & 0.00 \\
\hline & PPR & 2 & 20.00 & 2 & 100.00 & 0 & 0.00 & - & - & - & - & - & - & - & - & - & - & - & - \\
\hline & P. Total & 4 & 40.00 & 1 & 25.00 & 3 & 75.00 & - & - & - & - & - & - & - & - & - & - & - & - \\
\hline & Total & 10 & 100.00 & 5 & 50.00 & 4 & 40.00 & 0 & 0.00 & 0 & 0.00 & 1 & 10.00 & 0 & 0.00 & 0 & 0.00 & 0 & 0.00 \\
\hline \multicolumn{20}{|l|}{ Provincias } \\
\hline & Incrustaciones & 2 & 5.26 & 2 & 100.00 & 0 & 0.00 & 0 & 0.00 & 0 & 0.00 & 0 & 0.00 & 0 & 0.00 & 0 & 0.00 & 0 & 0.00 \\
\hline & Coronas & 5 & 13.16 & 3 & 60.00 & 2 & 40.00 & 0 & 0.00 & 0 & 0.00 & 0 & 0.00 & 0 & 0.00 & 0 & 0.00 & 0 & 0.00 \\
\hline & Puentes & 1 & 2.63 & 1 & 100.00 & 0 & 0.00 & 0 & 0.00 & 0 & 0.00 & 0 & 0.00 & 0 & 0.00 & 0 & 0.00 & 0 & 0.00 \\
\hline & PPR & 15 & 39.47 & 10 & 66.67 & 5 & 33.33 & - & - & - & - & - & - & - & - & - & - & - & - \\
\hline & P. Total & 15 & 39.47 & 10 & 66.67 & 5 & 33.33 & - & - & - & - & - & - & - & - & - & - & - & - \\
\hline & Total & 38 & 100.00 & 26 & 68.42 & 12 & 31.58 & 0 & 0.00 & 0 & 0.00 & 0 & 0.00 & 0 & 0.00 & 0 & 0.00 & 0 & 0.00 \\
\hline
\end{tabular}

n: Frecuencia absoluta.

$\%$ : Frecuencia relativa.

Prueba de Chi-cuadrado: Procedencia vs. Tipo de tratamiento $(\mathrm{p}=0.09)$ y Procedencia vs. $\mathrm{N}^{\circ}$ de tratamientos $(\mathrm{p}=0.59)$. 
Tabla 7. Tipo de tratamientos prostodónticos según género y procedencia en pacientes AM realizados en el Servicio de EPE del CDC-UPCH

\begin{tabular}{|c|c|c|c|c|c|c|c|c|c|c|c|c|c|c|c|}
\hline \multirow{3}{*}{ Sexo } & \multirow{3}{*}{$\begin{array}{c}\text { Tipo de } \\
\text { tratamiento }\end{array}$} & \multirow{3}{*}{$\mathbf{n}$} & \multirow{3}{*}{$\%$} & \multicolumn{12}{|c|}{ Procedencia } \\
\hline & & & & \multicolumn{2}{|c|}{ Lima Norte } & \multicolumn{2}{|c|}{ Lima Centro } & \multicolumn{2}{|c|}{ Lima Este } & \multicolumn{2}{|c|}{ Lima Sur } & \multicolumn{2}{|c|}{ P.C. del Callao } & \multicolumn{2}{|c|}{ Provincia } \\
\hline & & & & $\mathbf{n}$ & $\%$ & $\mathbf{n}$ & $\%$ & $\mathbf{n}$ & $\%$ & $\mathbf{n}$ & $\%$ & $\mathbf{n}$ & $\%$ & $\mathbf{n}$ & $\%$ \\
\hline \multicolumn{16}{|l|}{ Masculino } \\
\hline & $\begin{array}{l}\text { Incrustacio- } \\
\text { nes }\end{array}$ & 3 & 5.26 & 2 & 66.67 & 0 & 0.00 & 0 & 0.00 & 0 & 0.00 & 0 & 0.00 & 1 & 33.33 \\
\hline & Coronas & 10 & 17.54 & 4 & 40.00 & 0 & 0.00 & 3 & 30.00 & 0 & 0.00 & 1 & 10.00 & 2 & 20.00 \\
\hline & Puentes & 2 & 3.51 & 2 & 100.00 & 0 & 0.00 & 0 & 0.00 & 0 & 0.00 & 0 & 0.00 & 0 & 0.00 \\
\hline & PPR & 22 & 38.60 & 10 & 45.45 & 5 & 22.73 & 1 & 4.55 & 0 & 0.00 & 1 & 4.55 & 5 & 22.73 \\
\hline & P. Total & 20 & 35.09 & 10 & 50.00 & 3 & 15.00 & 3 & 15.00 & 0 & 0.00 & 0 & 0.00 & 4 & 20.00 \\
\hline & Total & 57 & 100.00 & 28 & 49.12 & 8 & 14.04 & 7 & 12.28 & 0 & 0.00 & 2 & 3.51 & 12 & 21.05 \\
\hline \multicolumn{16}{|l|}{ Femenino } \\
\hline & $\begin{array}{l}\text { Incrustacio- } \\
\text { nes }\end{array}$ & 9 & 6.29 & 3 & 33.33 & 2 & 22.22 & 0 & 0.00 & 0 & 0.00 & 3 & 33.33 & 1 & 11.11 \\
\hline & Coronas & 20 & 13.99 & 13 & 65.00 & 2 & 10.00 & 1 & 5.00 & 1 & 5.00 & 0 & 0.00 & 3 & 15.00 \\
\hline & Puentes & 6 & 4.20 & 3 & 50.00 & 1 & 16.67 & 0 & 0.00 & 1 & 16.67 & 0 & 0.00 & 1 & 16.67 \\
\hline & PPR & 57 & 39.86 & 37 & 64.91 & 8 & 14.04 & 1 & 1.75 & 0 & 0.00 & 1 & 1.75 & 10 & 17.54 \\
\hline & P. Total & 51 & 35.66 & 28 & 54.90 & 6 & 11.76 & 1 & 1.96 & 1 & 1.96 & 4 & 7.84 & 11 & 21.57 \\
\hline & Total & 143 & 100.00 & 84 & 58.74 & 19 & 13.29 & 3 & 2.10 & 3 & 2.10 & 8 & 5.59 & 26 & 18.18 \\
\hline
\end{tabular}

n: Frecuencia absoluta.

$\%$ : Frecuencia relativa.

Prueba de Chi-cuadrado: Procedencia vs. Tipo de tratamiento $(\mathrm{p}=)$ y sexo

\section{DISCUSIÓN}

Nuestro estudio mostró que la mayoría de pacientes AM eran de sexo femenino $(69,44 \%)$, asemejándose a los resultados de Medrano et al., en Chile, donde obtuvieron que la mayoría de su población de AM portadores de prótesis dental era de sexo femenino (12); y en Perú con los resultados de Vargas, obteniendo dentro de su muestra de AM a $87 \%$ del sexo femenino con prótesis dental (13).

Con respecto al grupo etario nuestros resultados mostraron que la mayor cantidad de pacientes fueron del grupo de viejos-viejos concordando con Yen et al., donde obtuvieron un promedio de edad de 76 años de AM que usaban prótesis dentales (14).

En relación a la procedencia se obtuvo que acudieron más AM provenientes de Lima Norte, coincidiendo con los resultados estadísticos del Instituto Nacional de Estadística e Informática (INEI) en el 2015 donde muestran que la mayor cantidad de AM se encuentran en dicha área, específicamente en San Juan de Lurigancho con un $83.9 \%$ y San Martín de Porres con $74,1 \%(15)$.

Con respecto a los tratamientos, las prótesis removibles se realizaron en un $75 \%$ frente a la prótesis fija con $25 \%$, siendo la PPR fue la que predominó, seguido de la PT. Cappo et al., en Brasil obtuvieron similares resultados en cuanto a que el menor tratamiento realizado fue la prótesis fija con $13.8 \%$. Sin embargo, difiere en cuanto a las prótesis removibles pues obtuvieron que la PT superó con 52,2\% a la PPR con $12,9 \%$ (16). A nivel nacional, Villareal, obtuvo como resultado, de una muestra de $177 \mathrm{AM}$, que el 50,8\% era edéntula y que la mayoría presentaba PT, $51 \%$ en 
la maxila y $37 \%$ en la mandíbula frente a la PPR con $11 \%$ en maxila y $13 \%$ en mandíbula (17).

Con respecto a los tipos de tratamiento se evidenció que el sexo femenino fue el grupo que más tratamientos se realizó. Similares resultados obtuvieron Mesas (18), Mallmann (19) y Barnard (20) en donde el mayor porcentaje de prótesis lo presentaban los AM del sexo femenino, y a nivel nacional, Peralta encontró que el sexo masculino es el que menos prótesis usa (21).

Según grupo etario, los viejos-viejos, se realizaron más tratamientos que los viejos-jóvenes, esto debido a que en nuestra muestra obtuvimos más pacientes de 75 a 89 años. Con respecto a las prótesis fijas, investigaciones de De-Andrade (22) y Borges (23) demuestran que estos tratamientos están relacionados a edades más jóvenes, coincidiendo con nuestro estudio, debido a que ese grupo poblacional presentan menos índice de edentulismo. En cuanto a las prótesis removibles, el grupo de los viejos-viejos fueron los que mayormente se realizaron PPR debido a un mayor índice de edentulismo que los viejos-jóvenes, pero menos que la de los viejos-longevos.

En relación a la procedencia, se dividió Lima Metropolitana según sus áreas obtenidas del INEI (24), en donde obtuvimos más personas provenientes de Lima Norte, específicamente de San Martín de Porres, debido a que dicha zona se encuentra más cercana al centro por lo que es más accesible para estas personas atenderse allí. Asimismo otros estudios indican que otro factor que influye en los tratamientos protésicos a elegir es el nivel socio económico, pues como se puede observar en nuestros resultados, en sectores como Lima Sur y Lima Este hay una mayor tendencia al uso de prótesis fija, pues según los resultados de la Asociación Peruana de Empresas de Investigación de Mercados (APEIM), hay un mayor nivel socioeconómico en distritos como San Isidro, Miraflores, San Borja, pertenecientes de Lima Centro, Este y Sur (25), por lo que ellos pueden acceder a estas opciones de tratamientos que son un poco más costosas.

No obstante, una de las limitaciones de esta investigación fue que la muestra no fue homogénea en cuanto a género. Además, algunos datos en las historias clínicas no estaban completos por lo que no se ha podido utilizar otras variables con lo cual hay un número reducido de muestra. Asimismo, la literatura no rescata muchos estudios similares sobre la determinación de tratamientos prostodónticos en $\mathrm{AM}$, por lo que esto repercute negativamente en la discusión de los resultados de esta investigación. Por ello se sugiere que a partir de esta investigación se puedan realizar más estudios que comparen otras variables como la necesidad de tratamiento con los tratamientos ya realizados y poder evaluar los motivos por los cuales la rehabilitación oral está tan abandonada en estos pacientes. De igual forma se puede tomar de referencia los resultados obtenidos y determinar los tipos de tratamientos prostodónticos en AM según enfermedades sistémicas más prevalentes para así dar hincapié en la importancia de realizar prótesis adaptándolos a su condición sistémica.

\section{CONCLUSIONES}

Las prótesis removibles fueron mayoritariamente realizadas con respecto a las de tipo fija, siendo la prótesis parcial removible la que más se evidenció en cada grupo etario, excepto en el de viejos - longevos.

El sexo femenino fue el que más recibió un tratamiento prostodóntico, en su mayoría prótesis parcial removible y en su defecto opuesto la prótesis fija, aunque la muestra no fue heterogénea, cabe resaltar que este grupo tiene mayor predisposición a la pérdida de piezas dentarias por factores culturales y socioeconómicos.

El lugar de procedencia que más se registró entre los pacientes adultos mayores fue el de Lima Norte, concluyendo que la cercanía a la clínica donde se obtuvo la muestra jugó un rol importante con respecto a la preferencia de atención por parte de los pacientes.

El presente estudio al ser el primero que denote la realización de tratamientos prostodónticos en pacientes adultos mayores, servirá para futuras investigaciones que puedan combinar otras variables como las comorbilidades que este grupo de pacientes usualmente presentan.

\section{Correspondencia:}

Sandro Lévano Loayza

Correo electrónico: sandro.levano.1@gmail.com 


\section{REFERENCIAS BIBLIOGRÁFICAS}

1. Ibérico L. Ley de la persona adulta mayor. Lima: Diario El Peruano; 2016.

2. Ribera JM. Características generales del paciente geriátrico.Clínicas Urológicas de la Complutense.1993;2:13-23.

3. Hernández R, Mendoza V, Martínez I, Morales L. Odontogeriatría y gerontología. México: Trillas; 2011.

4. Landinez N, Contreras K, Castro A. Proceso de envejecimiento, ejercicio y fisioterapia. Rev Cubana Salud Pública. 2012;38(4): 562-580.

5. Belando M. Cambios físicos y funcionales en el proceso de envejecimiento. ¿Es posible una calidad de vida en la vejez a través de programas socioeducativos? Cuestiones Pedagógicas. Revista de Ciencias de la Educación. 2001.

6. Owens D, Kalra S, Sahay R. Endocrinología Geriátrica. Rev Endocrinología y Metabolismo India. 2011; 15(2): 71-72.

7. Martínez C, Pérez V, Carballo M, Larrondo J. Polifarmacia en adultos mayores. Rev Cubana MGI. 2006;21(1-2).

8. Chávez B, Manrique JE, Manrique JA. Odontogeriatría y gerodontología: el envejecimiento y las características bucales del paciente adulto mayor: Revisión de literatura. Rev Estomatol Herediana. 2014;24(3):199-207

9. Duarte-Tencio T, Duarte-Tenorio A. Consideraciones en el Manejo Odontológico del Paciente Geriátrico. Rev Cient Odontol de Costa Rica.2012;8(1):45-54.

10. Haya MC, Blasco I, Cabo MB. La atención odontológica del paciente geriátrico con deterioro cognitivo. Avances en Odontoestomatolgia. 2015; 31 (3): 117-27.

11. Castellanos JL, Díaz LM, Lee E. Medicina en Odontología: Manejo dental de pacientes con enfermedades sistémicas. 3ra edición. México: El Manual Moderno; 2015.

12. Medrano A, Ruiz-Calixto B, Gutiérrez C. Impacto en la calidad de vida relacionada con salud bucal de la rehabilitación protésica convencional en pacientes desdentados completos, atendidos en el servicio público chileno. Rev Científica Mundo de la Investigación y el Conocimiento. 2017;1(4): 960-82.

13. Vargas K. Calidad de vida y salud bucal en usuarios de prótesis dental removible del centro integral del adulto mayor en Lima 2018. Tesis de Título profesional. Lima, Perú. Universidad Privada Norbert Wiener. 2018.

14. Yen YY, Lee HE, Wu YM, Lan SJ, Wang WC, Du JK, Huang ST, Hsu KJ. Impact of removable dentures on oral health-related quality of life among elderly adults in Taiwan. BMC Oral Health. 2015;1(5):15:1. doi: $10.1186 / 1472-6831-15-1$
15. Instituto Nacional de Estadística e Informática. Estadísticas de Personas adultas mayores. Lima: Instituto Nacional de Estadística e Informática;2015.

16. Cappo V, Sampaio E, Borgato M, Moura-e -Silva P, Nader S. El impacto de las condiciones bucales en la calidad de vida de personas con cincuenta o más años de vida. Ciencia y Salud Colectiva 2010; 15 (4): 2165-72.

17. Villareal L. Impacto de la salud bucal en la calidad de vida del adulto mayor en el centro de atención residencial geronto geriátrico "Ignacia Rodulfo Vda. De Canevaro" año 2011. Tesis de Maestría. Lima, Perú. Universidad Peruana Cayetano Heredia. 2012.

18. Mesas AE, Andrade SM, Cabrera MAS. Condiciones de salud bucal de ancianos de la comunidad urbana de Londrina, Paraná. Rev Bras Epidemiol. 2006; 9(4):471-80.

19. Mallmann FH, Toassi RFC, Abegg C. Perfil epidemiológico do uso e necessidade de prótese dentária em indivíduos de 50-74 anos de idade, residentes em três 'Distritos Sanitários' de Porto Alegre, Estado do Rio Grande do Sul, Brasil, em 2008. Epidemiol Serv Saúde. 2012; 21(1):79-88.

20. Barnard B, Vettori A, Ceriotti R. Perfil epidemiológico do uso e necessidade de prótese dentária em usuários de uma Unidade de Saúde da Família de Porto Alegre, Rio Grande do Sul, Brasil. Arq Odontol Belo Horizonte. 2014; 50 (4): 170-7.

21. Peralta F. Necesidad y situación de prótesis dentales en pacientes adultos que acuden a la clínica dental docente de la UPCH de julio a setiembre en el año 2015. Tesis de Título Profesional. Lima, Perú. Universidad Peruana Cayetano Heredia. 2017.

22. De Andrade C, Alves E, Faria T, Rocha S, de Souza C, Borie E, Corrêa Y. Perfil de los pacientes rehabilitados por medio de prótesis atendidos en el servicio público de salud en Ribeirão Preto, Brasil. Rev Odontoestomatol. 2015;31(6):347-53.

23. Borges CM, Campos ACV, Vargas AMD, Ferreira EF. Perfil de las pérdidas dentarias en adultos según el capital social, características demográficas y socioeconómicas. Cienc salud colectiva. 2014;19(6):1849-58.

24. Instituto Nacional de Estadística e Informática. XI Censo de población y VI de vivienda 2007. Lima: Instituto Nacional de Estadística e Informática;2007

25. Asociación Peruana de Empresas de investigación de mercados. Niveles socioeconómicos 2018.Lima: Asociación Peruana de Empresas de investigación de mercados;2019.

Recibido: 30-01-20

Aceptado: 20-09-20 